\title{
A ROBUST ORGANIZATIONAL POLICY FOR TASK AND RESOURCE ALLOCATION: A NOVEL FRAMEWORK
}

\author{
Shueb Ali Khan \\ Department of CSE, Shri Venkateshwara University, Gajraula, India. \\ Vinit Kumar Sharma \\ Department of Mathematics, Shri Ram College, Muzaffarnagar, India
}

\begin{abstract}
In a Multi-Agent System existing formalisms for implementing organizational policies assign specific roles to each agent. Examples are hierarchical organization, contract net protocol, social reasoning mechanism, and the use of matchmaker agents. These policies allow the problem solving roles of the agents to change dynamically but do not adapt to variations in computational load on the multi-agent system. They are designed to operate for a predefined maximum problem-solving load and fail to respond, when the number of task requests, exceed this limit.

This paper proposes an adaptive organizational policy, called E-RTA (Efficient Resource and Task Allocation), for Multi-Agent-Systems that operate under time constraints and varying computational loads. We consider MAS (Multi-AgentSystems) as consisting for several problem-solving organizations where each organization is comprises multiple agents that may be grouped into teams for specific problem solving.
\end{abstract}

Keywords: Multi Agent System, MAS, Resource Allocation, Organizational Polices.

Cite this Article: Shueb Ali Khan and Vinit Kumar Sharma, A Robust Organizational Policy for Task and Resource Allocation: A Novel Framework, International Journal of Computer Engineering and Technology, 10(2), 2019, pp. 31-40.

http://iaeme.com/Home/issue/IJCET?Volume=10\&Issue=2

\section{INTRODUCTION}

The rapid proliferation of multi-agent system applications, like management of networks for electricity transport and telecommunication, in factories to control manufacturing processes, in the medical domain for monitoring the condition of patients in an ICU, etc, places increased demands on the multi-agent system builder [1]. The multi-agent systems of today are dynamic and unpredictable. The type and frequency of requests in these applications varies non-deterministically. Hence it is becoming increasingly important to address the issue of adaptability of multi-agent systems to changing environmental conditions. 
Multi-agent system technology is rapidly passing the purely scientific stage and entering the market, where it is being used to solve real world problems in a range of industrial, commercial and medical applications. The main areas in which multi agent systems are being used include the following [1].

- Real-time monitoring and management of telecommunication networks, where agents are responsible, for call forwarding and signal switching and transmission

- Improving the flow of air traffic, where agents are responsible for interpreting data arising at different sensor stations.

- Monitoring and diagnosing faults in process control applications like nuclear power plants, where agents detect and diagnose faults.

- Electricity transportation management, where agents are involved in the diagnosis of disturbances in electricity transportation networks and their restoration.

- Information handling in extensive information flow like the Internet, where multiple agents are responsible for information filtering and gathering.

- Management of patient's health in a surgical intensive care unit (SICU), where agents monitor the condition of patients for occurrence of abnormal conditions and respond quickly to critical events.

- Electronic commerce and electronic markets, where 'buyer' and 'seller' agents purchase and sell goods on behalf of their users.

This rapid proliferation of multi-agent system applications places increased demands on the multi-agent system builder. The multi-agent systems of today are expected to operate in increasingly complicated environments that are dynamic and unpredictable.

But it is necessary to pose the following challenges to the multi-agent system builder.

- Agents must be able to

- adapt to unpredictable changes in problem solving environment, for instance when new information becomes available, it may invalidate existing beliefs or goals and

$\circ$ focus on higher priority tasks.

- The multi-agent system must be able to

- adapt to changes in load by diverting resources where they are needed most and

$\circ$ add new agents for problem solving in an incremental manner and thereby reorganize itself dynamically.

The rest of this paper is organized as follows. Section 1 gives a brief introduction of Multi Agent System and areas of application as Introduction. Section 2 gives the existing solutions as Related Work, Section 3 describes proposed model. We conclude our work in section 4 with future scope.

\section{RELATED WORK}

This section lists the important characteristics of multi-agent systems and provides a survey of work related to our aspire research. Section 2.1 explains the important characteristics of mufti-agent systems. Section 2.2 explores some of the existing mechanisms for implementing organizational policies. These methods emphasize the role of communication in achieving cooperation through organizational policies. 


\subsection{Characteristics of Multi-Agent systems}

One of the main concerns of multi-agent system research is to coordinate intelligent behavior among a collection of autonomous intelligent agents. The focus of this research is on developing mechanisms by means of which agents can be made to coordinate with each other for joint problem solving. Agents must be able to define their own goals and plans and perform complex interactions with other agents. They should be independent of any particular problem solving organization, and be able to define and change the organization as the problem solving activity evolves. This is possible only when agents are adaptive in nature. Most situations consist of a collection of agents with various skills including sensing, communication, planning and acting. Such groups possess the following characteristics $[2,3$, and 28]:

Openness: Agents in the system are not statically predefined, but dynamically enter and leave the organization. This necessitates mechanisms for locating agents. Locating an agent is a challenging task, especially in environments that include large numbers of agents and that have information sources, communication links, and/or agents that might be appearing or disappearing.

Autonomy: Each problem solver has to make local control decisions about what actions to execute with the information it has at hand when the decision is made. Agents need to coordinate their actions to promote beneficial interactions and avoid harmful ones because individual decisions have global Impacts.

Adaptability: Multi-agent systems are expected to operate in a constantly changing environment. These include a change in one or more of the following: The agent's environment, actions of other agents, availability of resources or problem solving demand on the organization.

The ability of the multi-agent system to adapt to such complex and dynamic environments by altering the problem-solving behavior of individual agents increases problem solving coherence.

Limited knowledge and perspective: These limit the capacity of an individual agent. This property of agents in called bounded-rationality [4] Bounded rationality is overcome by developing organizations of agents.

Shared limited resources: There are often shared limited resources with which an agent can execute tasks. This in turn leads to bounded rationality [4].

Differing agent capabilities: Agents typically differ in their appropriateness for a given task. The appropriateness of an agent for a task is a function of how well an agent's skills match the expertise required to do the task, the extent to which it.

This characterization leads to two main difficulties in the development of multi-agent systems [5]. First there are difficulties of optimal task assignment. Secondly, task coordination problems arise because tasks assigned to agents may not be independent. For instance, the execution of one task may facilitate or hinder the execution of another task.These difficulties can be overcome by choosing an effective organizational policy [6].

\subsection{Existing Mechanisms for Implementing Organizational Policies: Literature Review}

This section explores some of the existing mechanisms for implementing organizational policies. These methods emphasize the role of communication in achieving cooperation through organizational policies.

An organizational policy addresses the issues of task and resource allocation, which are problems of assigning responsibilities and resources for a sub-problem to a problem solver. A 
better alternative is to have an adaptive task allocation mechanism that assigns tasks in a dynamic and distributed way Individual agents decide what tasks they will take on and how they will cooperate to achieve a goal collectively. This results in proper load balancing and bottleneck avoidance.

Some of the existing formalisms for implementing organizational policies in multi- agent systems are hierarchical organization, contract nets, social reasoning mechanism, and the use of economic methods.

\subsubsection{Hierarchical Organization}

In a hierarchical organization, decision-making and control is concentrated in a single problem solver at each level in the hierarchy. Interaction is through vertical communication from superior to subordinate agent. The subordinate agents have no autonomy. It is the superior agents that exercise control over resources and decision- making [30, 29, and 7]. Hierarchical organizations are therefore not suited for autonomous agent interactions.

Kumar et al. in [36] proposed the agent based layered framework for resource allocation. The framework mainly depends on one of the information retrieval technique name called vector space model. The implementation shows by using vector space model implementation as agent using JADE. The two vectors (Query and Total Availability of resources) are given as input and the framework provides the similarity coefficient for the data centers related to specific query.

\subsubsection{Contract Net Protocol}

Contract net protocol $[8,31,9]$ achieves opportunistic and adaptive task allocation among a collection of problem solvers using a framework called negotiation based on task announcement, bids, and awarded contracts Is offers symmetry in the transfer of control process by using an interactive mutual selection (negotiation) process where the caller evaluates potential respondents from its perspective (via the bid evaluation process) and the respondents evaluate the available tasks from their perspective (via the task evaluation process) The evaluation procedures are local to the agents doing the evaluation.

Two kinds of agents, manager and contractor, exist in contract net protocol Managers announce tasks to contractors, which undertake them. When the manager has a task, it announces it to all contractors within the system Each contractor selects form the announced tasks, the one that best matches its own capabilities and bids for it. Finally the manager chooses what it believes to be the most appropriate bid and award the task to that agent There may exist multiple managers and contractors in the system simultaneously. A manager can make multiple announcements simultaneously while a contractor can bid for only one task at a time.

Cheng and Ishida [8] investigated the various effects of mutual selection mechanisms on manager and contractor utilities, Their analysis (based on queuing theory) produced the following conclusions.

Sandholm [10] describes a variant of the contract net protocol for e-commerce in which tasks can be clustered to allow individual agents to bid for complementary tasks as bundle further extensions were made by Sandholm and Lesser [11,12] where decornmitments and decommitment penalties were introduced.

Other applications of contract net protocol includes Enterprise [13], the transportation control system TRACONET [10] and factory floor operations [14].

\subsubsection{Social Reasoning Mechanism (SRM)}

This model is based on social power theory $[15,16]$. In the SRM model [17,18, 19,20,21,22] dynamic coalitions are formed on the basis of motivation in the form of social dependence 
relations. Coordination starts in a recognition stage. This stage is seen as the trigger of negotiation, in which agents assess the potential for cooperation by recognizing how they depend on each other.

In SRM, agents maintain models of acquaintances in a data structure called external description (ED). An ED contains the agent's goals, plans, actions, and resources.

The Mobile Agent is also an option to enhanced adaptability. The paradigm of mobile has two general goals: reduction of network traffic and load balancing. It is majorly being used in enhancement of telecommunication services $[32,33]$.

Agent cloning $[25,26]$ is also proposed as a more general approach to agent mobility. It is the act of creating and activating a clone agent to perform some of the agent's tasks for balancing the loads Cloning is performed when an agent predicts an overload, thus increasing the ability of the multi-agent system to perform tasks.

The review presents the state of the research in task, resource allocation frameworks, the methods for designing real-time agents, and methods for reorganizing the multi- agent system in response to computational overloads. In order to obtain a truly adaptive organizational policy we require a complete and comprehensive framework that does the following

- Considers criticality of tasks,

- Allows individual agents to be adaptive, and also

- Allows the multi-agent system to dynamically reorganize itself.

As agents operate in environments where they neither have complete nor correct beliefs about the environment / other agents, it is indeed essential for every agent to have the capability to engage in collaborative problem solving. However, as we are interested in situations with varying problem solving load, it is not enough if individual agents possess team rationality. There should also be a means of having the entire multi - agent system alter its organization in accordance with these variations and continue to provide services as per requirements, always giving preference to higher priority tasks in case of temporal conflicts A comprehensive framework that satisfies all the above requirements is not available.

\section{PROPOSED MODEL}

\subsection{Overview of Proposed Framework}

The existing policies allow the problem solving roles of the agents to change dynamically but do not adapt to variations in computational load on the multi-agent system. They are designed to operate for a predefined maximum problem-solving load and fail to respond, when the number of task requests, exceed this limit.

This limitation can be divided into the following two sub-problems.

- Allocation of tasks to agents within an organization through the tasks allocation policy, and

- Allocation of resources to each of these organizations, through the resource allocation policy

A decrease in the problem solving load will result in surplus resources. Hence, we need a mechanism for dynamic allocation of resources to the MAS to allow it to operate in unpredictable environments.

Our clear aim is to find a suitable / adaptive organization that exhibits high performance despite unanticipated changes in the environment (nature, type and frequency of requests) and time constraints.

In this sequence, we are proposing and adaptive organizational framework called E-RTA (Efficient Resource and Task Allocation). It would be the presumption that agents obey the responsibility code-of-conduct for joint activity [27]. The proposed framework will have 
several problem-solving organizations where each organization is comprised of multiple agents that may be grouped into teams for specific requests with an associated priority and deadline.

A request that arrives at an organization will be solved cooperatively by agents within that organization and independently of the other organizations. The rate of arrival of problem solving requests at each of these organizations varies with time. As a result, the requirement for resources also varies. At any particular instant, some organizations may have surplus resources, while others have insufficient resources and thereby turn down problem solving requests.

In order to minimize these lost requests, the allocation of resources to organizations needs to be changed dynamically. This reallocation results in reorganization of the multi-agent system and is intended to balance demand for resources at each organization with its supply.

A layered approach will be used for developing an adaptive organizational policy that simplifies the design of agents and to divide the problem into two sub-problems-

- Allocation of tasks to agents within an organization

- Allocation of agents (as resources) to each of these organizations

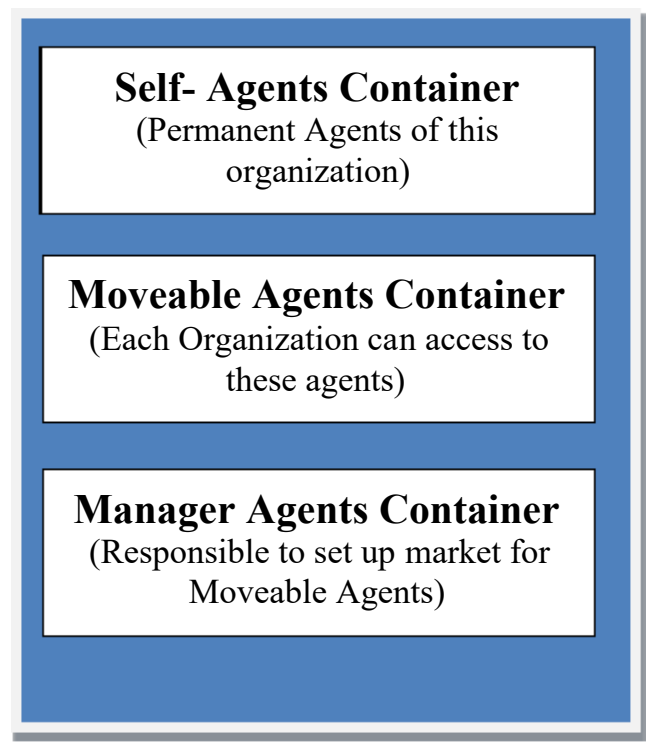

Figure 1. Outline Layered Design for a Single Adaptive Proposed Organization in E-RTA

The fig. 1 shows the Outline Layered Design for a Single Adaptive Proposed Organization, that contains three different types of agents, Self-Agents (Permanent Agents of an organization and always belongs to it), Moveable Agents (or Transferable agents, each Organization can access to these agents) and Manager Agents (Responsible to set up market for Moveable Agents).

\subsection{Operational Framework Design}

The operational framework organization for the multi-agent system with individual collaboration is demonstrated below. E-RTA based applications are intended for use in unpredictable environments. 


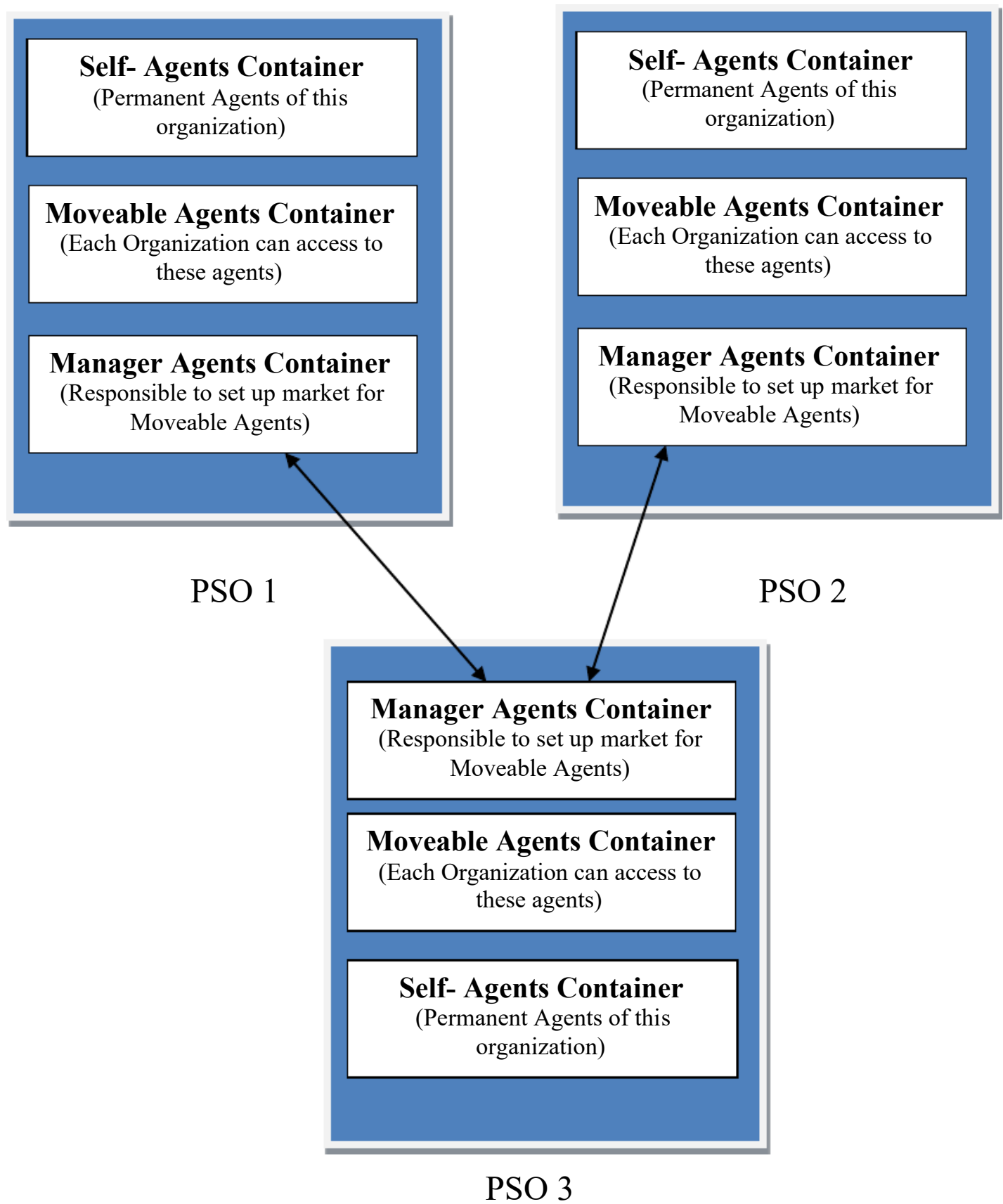

Figure 2 Outline Operational Framework Design for E-RTA

E-RTA agent architecture therefore likely to be allows the following to the agents-

- To adapt unpredictable changes in problem solving environment (by maintaining its beliefs and goals always constant with the latest information that it receives from the environment / other agents)

- To exhibit team rationality by means of the joint intention representation and the override mechanism, when the conditions for executing an intention no longer exist, the intention can be dropped through the override mechanism and fellow team members can be informed of this fact. This aids in time constrained problem solving by preventing agents from pursuing useless activities and reducing the amount of wasted effort 
- Focus on higher priority tasks, when an irregularity is detected between an existing intention and a new one, it would be resolved by the inconsistency resolver in favor of the higher priority intention.

In addition to this, the multi-agent system organization would facilitate cooperative problem solving among agents within an organization, and allows the multi- agent system-

- To adapt to changes in load by diverting resources where they are needed most,

- Addition of new agents for problem solving in an incremental manner to enhance a particular organization as well as the framework,

- the overall target would be reorganizing it dynamically,

\section{SUMMARY \& FUTURE SCOPE}

Existing methods for implementing organizational polices [34, 30, 18, 35, 29, 31], address only some of the issues in isolation and hence cannot meet all the requirements of dynamic adaptation. This proposal proposed a single comprehensive organizational policy that can operate under time constraints and varying computational loads.

The problem of designing such an organizational policy, we follow the layered approach, is divided into the following two sub-problems.

- Allocation of tasks to agents within an organization through the tasks allocation policy, and

- Allocation of resources to each of these organizations, through the resource allocation policy

\section{Future Scope}

In future, the framework can have the selective intelligence at individual agent level by having analytical / machine learning approach to enhance the self adaptation of each individual agents as well as the manager.

\section{REFERENCES}

[1] N R Jennings, Katia Sycara, Micheal Wooldridge, "A Roadmap of Agent Research and Development", Autonomous Agents and Multi Agent Systems, 1, DO 275-306. 1998.

[2] K P Sycara and D Zeng, "Coordination of Multiple Intelligent Software Agents", International Journal of Intelligent and Cooperative Information Systems, 5, 2\&3, pp 181211, 1996.

[3] K P Sycara, K Decker, A Pannu, M Williamson and D Zeng, "Distributed Intelligent Agents", IEEE Expert, 11, 6, pp 36-46, December 1996.

[4] Jon Doyle, "Rationality and its Roles in Reasoning", in Proc of Eighth National Conf. On AJ, pp 1093-1100, 1990.

[5] AH Bond and L Gasser (eds), "Readings in Distributed Artificial Intelligence", Morgan Kaufmann, 1988.

[6] Stephanie Cammarata, David Mc Arthur, Randall Steeb, "Strategies for Cooperation in Distributed Problem Solving", Proc. of Eighth International Conference on AI, August, pp 767-770, 1983.

[7] L D Erman and V R Lesser, "A Multi Level Organization for Problem Solving Using Many Diverse Cooperating Sources of Knowledge", Proc. of International Conference on AI, pp 483-490, 1975. 
A Robust Organizational Policy for Task and Resource Allocation: A Novel Framework

[8] Cheng Gu, T Ishida, "Analyzing the Social Behavior of Contract Net Protocol", LNAI, 1038 (Berlin: Springer), pp 116-127, 1996.

[9] R G Smith, R Davis, "Frameworks for Cooperation in Distributed Problem Solvers", IEEE Transactions on Systems, Man, and Cybernetics ,11,1, pp 61-70, 1981.

[10] T W Sandholm, "An Implementation of the Contract Net Protocol based on Marginal Cost Calculations", AAA1-93, pp 256-262, 1993.

[11] Sandip Sen and Edmund H Durfee, 'The Role of Commitment in Cooperative Negotiation", International Journal of Intelligent and Cooperative Information Systems, 3,1, pp 67-81, 1994.

[12] T W Sandholm, V R Lesser, "Advantages, of Leveled Commitment Contracting Protocol", Tech Report 95-72, Univ. of Mass, Sep 1995.

[13] T W Malone, R E Fikes, K R Grant, M T Howard, "Enterprise: A Market- like Task Scheduler for Distributed Computing Environments", The Ecology of Computation, North Holland, pp 177-205,1988.

[14] H Van Dyke Parunak, "DAI and Manufacturing Control Some Issues and Insights", Decentralized AJ: Proc of First European Workshop on Modeling.

[15] Cristiano Castelfranchi, "Social Power: A Point Missed in Multi-Agent, DAI, and HCl" In Yves Demazeau \& Jean Pierre Muller, editors, Decentralized AI, pp 49-62, Elsevier Science Publishers, 1990.

[16] Cristiano Castelfranchi, Maria Micelli, Amedo Cesta, "Dependence Relations Among Autonomous Agents", In Eric Werner \& Yves Deamzeau, editors Decentralized AI, 3, pp 215-227, Elsevier Science Publishers, 1992.

[17] Jaime S Sichman, "DEPINT : Dependence-Based Coalition Formation in an Open MultiAgent Scenario", Journal of Artificial Societies and Social Simulation, 1, 2, March 1998.

[18] J S Rosenchien, "Synchronization of Multi-Agent Plans", Proc 1st National Conference on AI, ppl 15-119, 1982.

[19] Jaime Sichman and Yves Demazeau, "Exploiting Social Reasoning to Deal with Agency Level Inconsistency", In Proceedings of First International Conference on Multi Agent Systems, (ICMAS-95), San Fransisco, USA, June 1995.

[20] Jaime Sichman and Yves Demazeau, "Using Class Hierarchies to Implement Social Reasoning in Multi Agent Systems", In Proceedings of 11th Brazilian Symposium on Artificial Intelligence, (SBIA-94), Fortaleza, Brazil, Outubro, 1994.

[21] Jaime Sichman,R Conte,Y Demazeau,C Castelfranchi ,"ASocial easoning Mechanism Based on Dependence Networks", in Proc. of 11 European Conf. On AI, Amsterdam, pp 188-192, 1994.

[22] Jaime Simao Sichman, Yves Demazeau, "A Model for the Decision Phase of Autonomous Belief Revision in Open MAS", Journal of Brazilian Computer Society, 31, pp 40-50, 1996.

[23] K S Decker, K Sycara and M Williamson, "Cloning for Intelligent Adaptive Information Agents", in C Zhang and D Lukose (eds), Multi Agent Systems. 
[24] O Shehory, K Sycara, P Chalasani, S Jha , "Agent Cloning: An Approach to Agent Mobility and Resource Allocation", IEEE Communications 36, 7, pp 58-67, July 1998.

[25] N R Jennings, "Joint Intentions as a Model of Multi-Agent Cooperation in Complex Dynamic Environments", Ph.D. Thesis, Dept. of Electronic Engineering, Queen Mary and Westfield College, University of London, 1992.

[26] Luc Steels, "Cooperation Between Distributed Agents Through Self Organization", in Demazeau Y, Muller JP, editors, Decentralized AI, Proc of First European Workshop on Modeling Autonomous Agents in Multi Agent World (MAAMAW-89), pp 175-196, Elsevier Science Publishers, 1990.

[27] K P Sycara, "Multiagent Systems", AI Magazine, Summer 1998, pp79-92, 1998.

[28] Daniel D Corkill, "Hierarchical Planning in a Distributed Environment", In Proceedings of Sixth International Joint Conference on Artificial Intelligence, pp 168-175, Cartridge, Massachusetts, August 1979.

[29] R G Smith, "Contract Net Protocol High Level Communication and Control in Distributed Problem Solvers", IEEE Transactions on Computers, 29,12, pp 1104-1113, 1980.

[30] Ahmed Karmouch, "Mobile Software Agents for Telecommunications", Guest Editorial, IEEE Communications Magazine, pp 24-25, July 1998.

[31] Marie-Pierre Gervais, "Enhancing Telecommunications Service Engineering with Mobile Agent Technology and Formal Methods", IEEE Communications Magazine, pp 38-43, July 1998.

[32] Aure Anderson, F Ygge, "Managing Large Scale Computational Markets", http://www.enersearch. se/ ygge.

[33] James Kurose, Rahul Simha, "A Microeconomic Approach to Resource Allocation in Distributed Computer Systems", IEEE Transactions on Computers, 38, 5, pp 705-711, May 1989.

[34] Kumar A., Pilli E. S. and Joshi R. C., "An Efficient Framework for Resource Allocation in Cloud Computing," in 4th IEEE International Conference on Computing, Communication and Network Technologies (ICCCNT'13), Tamilnadu, India, 2013, pp. 1-6. 